\title{
A Simulation Technique for Wireless Mesh Networks to Present Its Topology and Evaluate Its Impact on Communication Revolution
}

\author{
Ahmed Alahdal ${ }^{*}$, G. N. Shinde ${ }^{2}$ \\ ${ }^{1}$ School of Computational Science, SRTM University, Nanded, India \\ ${ }^{2}$ SRTM University, Nanded, India \\ Email: *abahdal@gmail.com,shindeg@yahoo.co.in
}

Received 10 November 2015; accepted 26 February 2016; published 29 February 2016

Copyright (C) 2016 by authors and Scientific Research Publishing Inc.

This work is licensed under the Creative Commons Attribution International License (CC BY).

http://creativecommons.org/licenses/by/4.0/

c) (i) Open Access

\begin{abstract}
The topology of full Mesh has driven the Researchers in the field of wireless communication to claim that wireless mesh networks can be a significant change in the future of wireless communication. This paper presents a simulation process using Mesh routers which simulate wireless mesh network with multiple values to investigate its performance and how results can be optimized in such experience. During the process, we expand the area by assigning different values according to the number of routers needed for implementation. We create a proper centralized connectivity with more and less number of nodes using IEEE802.11a and $g$ as a backbone and source of internet. We also study and observe its topology which provides multiple interfaces and paths between endpoints as well can control, self heal, configure and organizes itself.
\end{abstract}

Keywords

WMNs, Wireless Communication, Mesh Router, IEEE802.11a \& g

\section{Introduction}

The need of wireless technology is increasing day by day creating difficulties on the internet providers to maintain the same efficiency and scalability, such increment requires development in the existing technologies or even adapting new once which can meet these demands. But what is more beneficial and flexible a wired net-

*Corresponding author.

How to cite this paper: Alahdal, A. and Shinde, G.N. (2016) A Simulation Technique for Wireless Mesh Networks to Present Its Topology and Evaluate Its Impact on Communication Revolution. Communications and Network, 8, 39-44.

http://dx.doi.org/10.4236/cn.2016.81005 
work or a wireless network, according to the improvement factors and daily needs for a faster world it has become clear that wireless network is more beneficial then wired network. Even thus transferring data wirelessly is one of the most challenging tasks in the field of communication, but it still exists and with the growth of demands and need to ensure more speed and capability, a number of researchers have been struggling to find the most efficient way of wireless communication. IEEE802.11 wireless technology has been serving the world by providing the best possible effort in the field of wireless communication (Wi-Fi, cellar, etc.) despite the fact that each day is a challenge to transfer an increased enormous number of data, but the more data we transfer the more load and traffic we face, which sometimes results in a data losses, data discard or even data failure. Wireless mesh network is believed to be the safest, fastest possible mechanism that would make the dream of seamlessly world come true. Wireless mesh network is considered as a new technology which can be adapted, "researchers claim". But how good wireless mesh network can be that is the main work for the researchers to prove. Wireless Mesh Networks (WMNs) offer low up-front cost, reliable coverage, easy network maintenance, dynamic self organization and self-configuration. Those characteristics are ideal for the next-generation wireless communication systems in terms of seamless broadband access. WMNs have therefore attracted more and more attention from both academia and industry in recent years. Different from the other wireless networks, most applications of WMNs are broadband services with heterogeneous quality of service (QoS) requirements (e.g., transmission rate, packet loss ratio and delay jitter) [1]. Wireless mesh network has many features that would make it a strong choice for the internet users or even providers. It is possible to equip each infrastructure node with multiple radios, and each radio is capable of accessing multiple orthogonal channels, referred as Multi-Radio Multi-Channel transmissions [2]. Furthermore wireless mesh network Signals cannot typically penetrate walls so Mesh routers have to be well implemented.

- The common standard IEEE802.11a with $5 \mathrm{GHz}$ and up to 54 Mpbs speed is a considerable source of internet for WMNs.

- The common standard IEEE802.11g with $2.4 \mathrm{GHz}$ and up to 54 Mpbs speed is considered only on short distance signals but it can also be an internet source as well.

Utilizing the IEEE802.11ac for the Wireless Mesh Network is possible as it has been announced but it is still not in use. The expected data transfer speed is believed to be faster than the fiber cable. The term "wireless mesh networks" describes wireless networks in which each node can communicate directly with one or more peer nodes [3].

\section{Background and Related Work}

The next generation wireless community networks are envisioned to provide high-speed and high-throughput connectivity for end users by leveraging a number of technologies, such as fourth/fifth generation (4G/5G) mobile cellular systems, IEEE-802.11-based wireless networks, and IEEE802.16 (WiMAX) broadband wireless networks, The WMN is one of the key components of such community networks [4]. Wireless Mesh Network (WMN) is a new type of broadband wireless access network that integrates the advantages of Wireless Local Area Network (WLAN) and Ad Hoc Network. This special network offers a high speed and enough capacity over large areas [5]. WMNs have become popular due to its universal networking capabilities. It supports a wide range of applications, such as public safety, emergency response communications, intelligent transportation systems, and community networks etc. It consists of two types of wireless nodes namely Mesh Routers and Mesh Clients. Each node does not operate as a host only but also acts as a router, which forwards the packets on behalf of other nodes that may not be within the direct transmission range of their destination [6]. Typically, the backbone of a wireless mesh network (WMN) is made up of dedicated wireless nodes called mesh routers (MRs), which are configured in an ad hoc mode and use unidirectional antennas, with one or multiple wireless radio interfaces based on IEEE802.11 technologies. These MRs can be freely organized into any network topology, and communicate with each other using protocols such as Optimized Link State Routing (OLSR) [7]. In addition to mesh networking among mesh routers and mesh clients, the gateway/bridge functionalities in mesh routers enable the integration of WMNs with various other networks [8]. The specific characteristics of WMNs, such as the stationary of MRs and high storage capacity compared to MCs, motivate us to utilize the MRs as replica servers. In view of this fact, content replication schemes can be used to reduce the data fetching delay and decrease the network load in WMNs [9]. Wireless mesh routers can be installed on rooftops, traffic lights, or street light poles to help each other forward data packets to or from vehicles, as well as from servers on the Internet 
[10]. However, these wireless solutions also have fundamental problems that their performance can be different depending on the dynamicity of the network environment. For example, WMNs deployed for public surveillance may suffer from various exterior interferences [11].

\section{Preview of the Proposed Design}

The design this paper presents is to simulate the wireless mesh network in order to understand the Mesh network topology. It is important to mention that routers should be central to the area being covered otherwise the user at the edge of the signal network will have slower speed comparing to the other users in the central area. During the simulation process we have observed the work of the network topology and how many routers (nodes) have been implemented. We have investigated the wireless mesh network to optimize the speed, availability and coverage. The source (backbone) node was providing the network access to the rest of the nodes as it is the only wired node in the network. Note, More than one backbone can provide the network access. The results and figures of this proposed design shall be discussed clearly in the simulation results section.

\section{Simulation Results}

In the following figures, we show the area and the number of routers (MR) and the signal each node can cover. We have simulated before it gets connected and after it is connected. We implemented the routers randomly but each of them is deployed within the reach of the other router. In Figure 1, we initiate the values; a total number of twenty Mesh routers (MR) in an assumed area of $10 \times 10$ meters, the signal range of each node planted covers an area of 10 meters, for such short distance we use the common standard IEEE802.11g which serves short distance range, as it is illustrated.

When routers (MR) get connected, each one of them engages with the nearest MR automatically and that is called self configuration, typically each MR is capable of getting connected with more than one source, referred to such process as a self administration. In the next figure (Figure 2), we illustrate how MR are connecting making the area totally covered with less possibility of having a network failure.

From the main router (node) we notice multiple connection made in different direction, we can see the MR connected to the nearest Mesh router (MR) providing a unique structure that shows how WMNs operate. Let's suppose one of the MR gets disconnected due to any reason, would the network fail, actually it will not because other MR will provide the coverage for the lost MR (self healing).
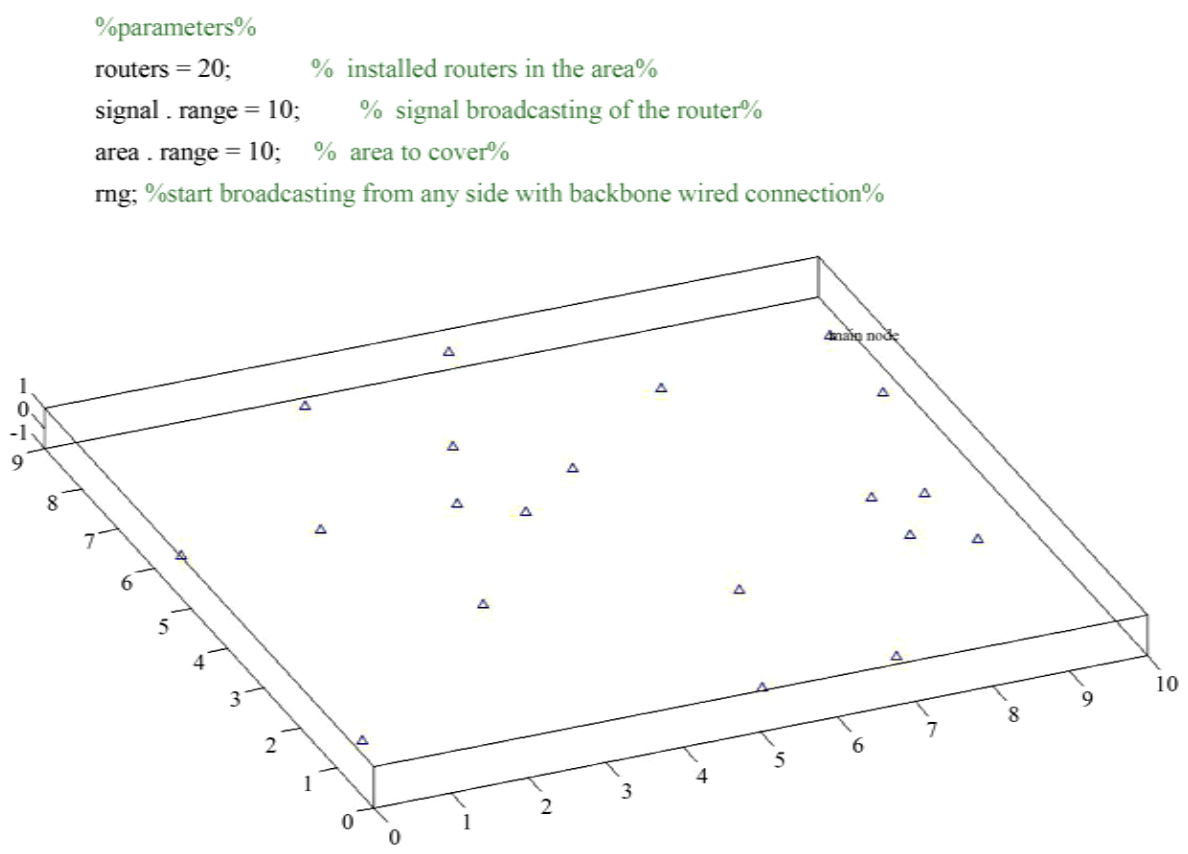

Figure 1. Shows the MR planted in the area but not yet broadcasting. 


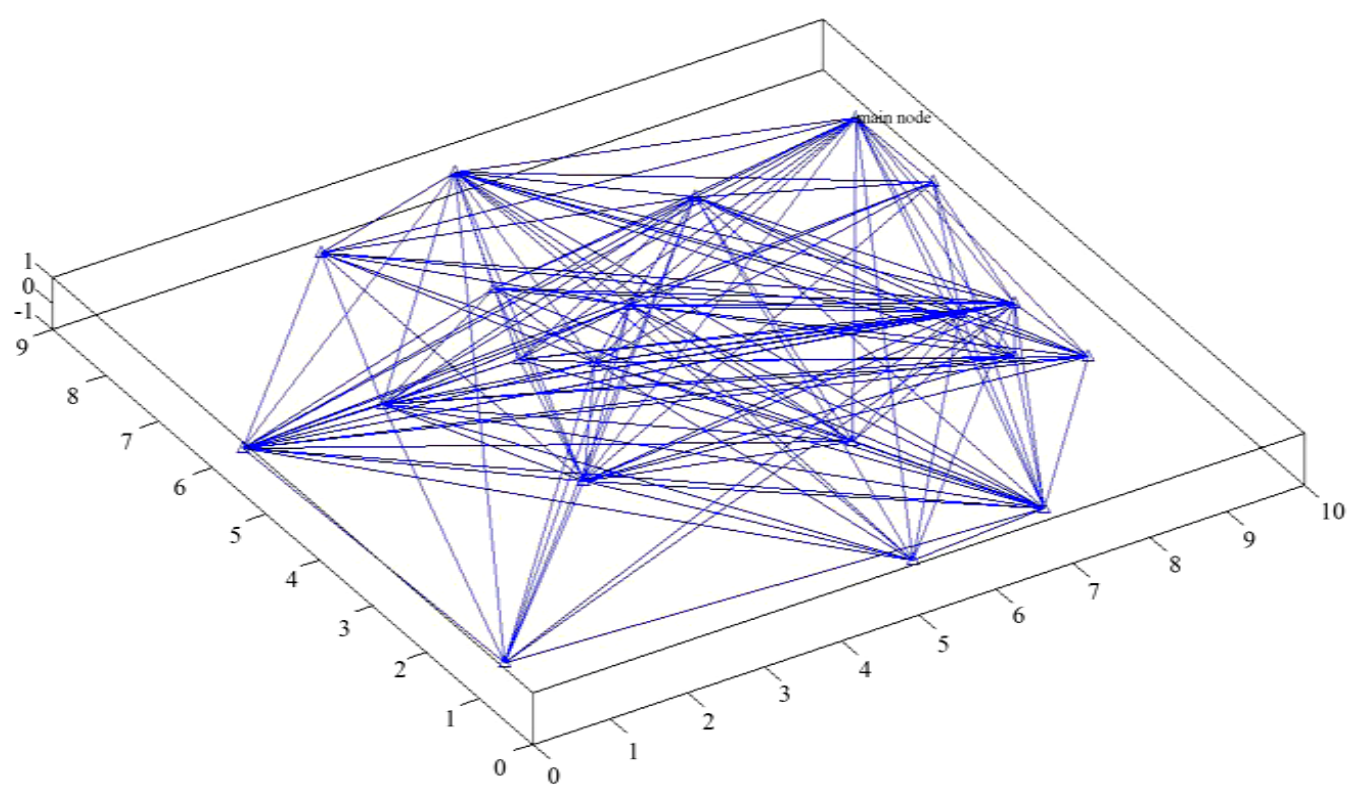

Figure 2. Shows the MR planted and broadcasting to each other.

In Figure 3: we have increased the number of nodes, the array to cover bigger place. We have planted a total number of hundred routers (MR) in an area of $100 \times 100$ meters with a signal range of 45 meters that uses the standard IEEE802.11a, with such increment routers (MR) will be examined to ensure its capacity. The reason for using the IEEE802.11a is that larger areas requires stronger signal broadcasting that would be able to reach long distance, Short signal range will not be sufficient even if the number of routers (MR) is increased.

As we can see in the above figure the routers (MR) were programmed to spread in a structure that allow us to cover the entire required area ensuring the availability of the wireless mesh network coverage. It is important for mesh routers to output more paths as well receives multiple interfaces and have a strong signal, so packets can travel through different paths which results in availing channels allocations and decreases the data failure of the packet also increases the throughput but for that to happen mesh routers should not be in the edge of the signal provided from other MR, it is advised to set all of the MR in a centralized area with respect to the signal range.

The way signals connecting the Mesh routers enlighten us with the fact that each MR (node) is capable of getting access to more than one source simultaneously as well it can receive signals from different sources.

\section{Figure Analysis}

$>$ In Figure 1, the structure of the network was organized, which means the distance of each node to the next node was calculated. The topology of the nodes allow them to determine the distance required from one point to the other to avail the connection among them and prevent signal failure among nodes while operating, this is called (self configuring).

$>$ In Figure 2, the signals have spread Hop by Hop to reach all nodes considering that each mesh router (node) is capable of configuring itself to join the other nodes and to complete the formulation of the network.

$>$ In Figure 3, we have extended the area that needs to be covered and assigned more values mesh router (node) to the network as well as long range signal nodes to satisfy the need for full coverage as a part of the simulation process.

$>$ In Figure 4, the signal range was extended also the mesh router (node) were increased even thus there was no nodes failure. But we noticed some MR less connected with others assuming that some of these MR (nodes) are planted opposite to a bridge or facing long building, etc.

In Table 1: we illustrate the simulated values we assigned to the simulation process we therefore presented the base for WMNs, speed and frequency bands. The table showed the approximate area that was covered, the Mesh routers in each area, and the signal of the Mesh router according to the need. For example if $100 \mathrm{MR}$ broadcast a signal less than 150 foot the area would not be completely covered also the signals would be weak 
$\%$ parameters $\%$

routers $=100 ; \quad \%$ installed routers in the area $\%$

signal . range $=45 ; \quad \%$ signal broadcasting of the router $\%$

area . range $=100 ; \quad \%$ area to cover $\%$

rng; \%start broadcasting from any side with backbone wired connection $\%$

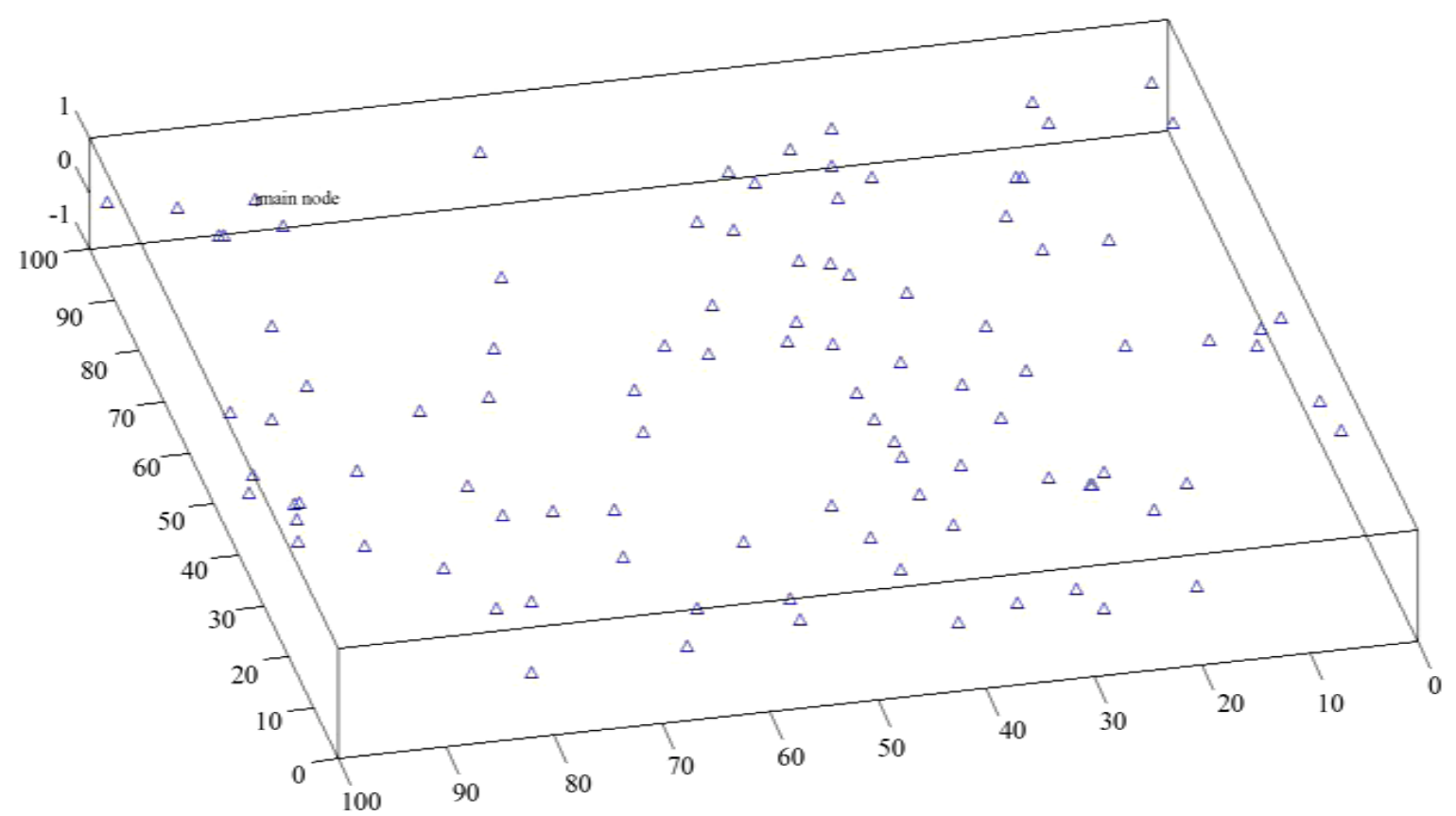

Figure 3. Shows larger area and an increased number of MR before broadcasting.

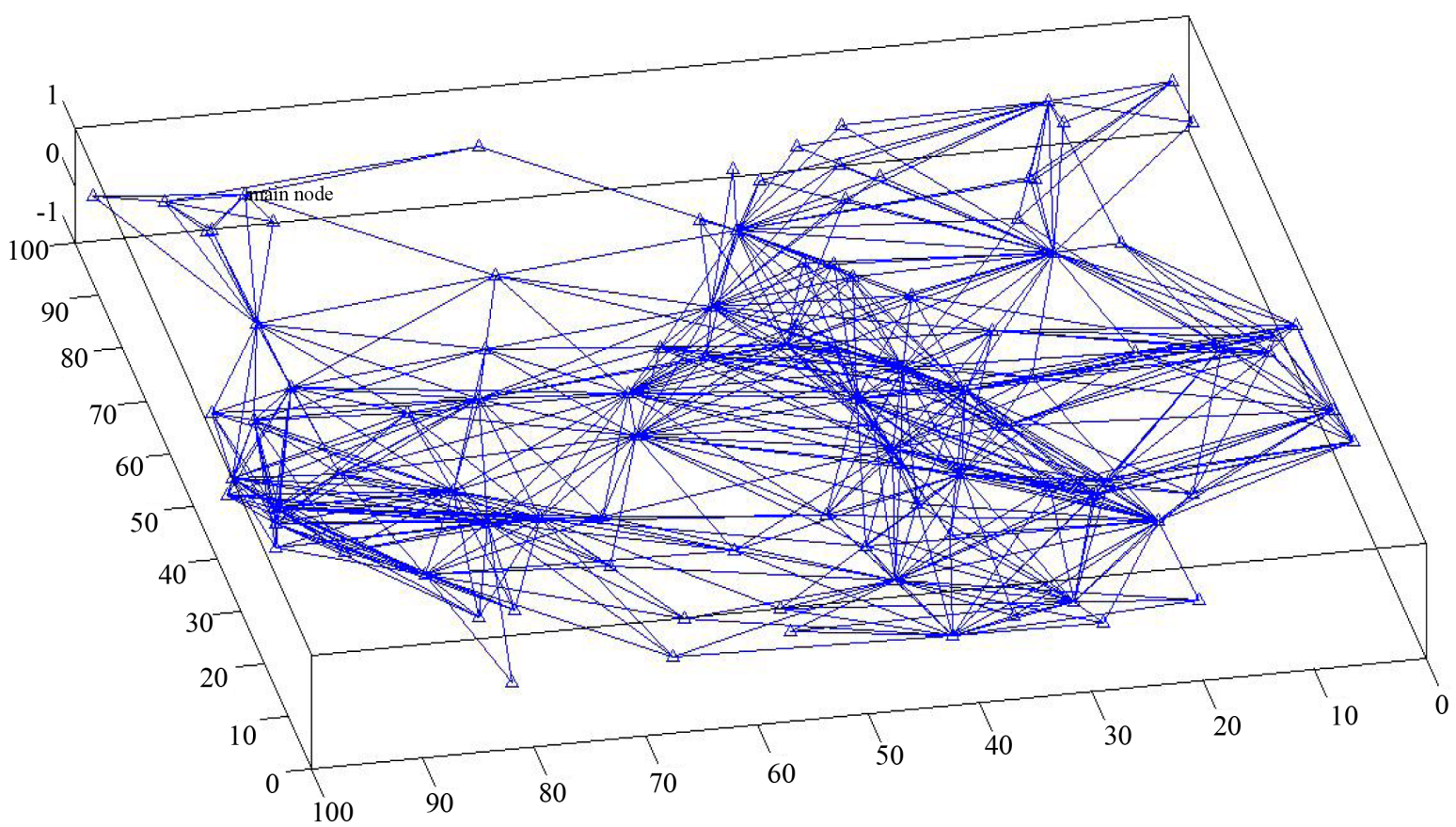

Figure 4. Shows MR when broadcasting in larger areas and with more MR. 
Table 1. Performance evaluation.

\begin{tabular}{|c|c|c|c|c|c|c|c|}
\hline Figure No. & $\begin{array}{l}\text { Base for } \\
\text { WMNs }\end{array}$ & Speed up to & $\begin{array}{c}\text { Freq bands } \\
\text { up to }\end{array}$ & $\begin{array}{l}\text { No. of mesh } \\
\text { routers }\end{array}$ & Approx area & $\begin{array}{l}\text { Signal } \\
\text { range }\end{array}$ & $\begin{array}{l}\text { Nodes } \\
\text { failure }\end{array}$ \\
\hline 1 & IEEE802.11g & 54 Mpbs & $\begin{array}{c}2.4-2.485 \\
\mathrm{GHz}\end{array}$ & 20 MR (nodes) & $10 \times 10 \mathrm{~m}$ & $\begin{array}{l}10 \mathrm{~m} \text { or } \\
33 \mathrm{foot}\end{array}$ & 0 \\
\hline 2 & IEEE802.11a & 54 Mpbs & $\begin{array}{c}5.1-5.8 \\
\mathrm{GHz}\end{array}$ & 100 MR (nodes) & $100 \mathrm{~m} \times 100 \mathrm{~m}$ & $\begin{array}{l}45 \mathrm{~m} \text { or } \\
150 \text { foot }\end{array}$ & 0 \\
\hline
\end{tabular}

which effect the strength of the rate transfer.

\section{Conclusion}

In this paper, we proposed a simulation process to illustrate the operational technique of Wireless Mesh Network; it showed flexibility to be expanded according to the values initiated as input data, taken into account the need for implementing some mesh router in a central area. We have utilized the common standard IEEE802.11a and g as a source of internet during the investigation. The network was assigned with multiple values such as, different number of mesh routers, scaling larger area and multiple interfaces with variant signals to broadcast wireless mesh network. The results we have had were promising considering the possibility of linking a town. We had also noted some nodes with fewer interfaces which of course were due to the non centralized positioning. Central area in the time of implementation was important to cover more of the area and provide alternative interfaces for each Mesh router. Finally, the entire simulation process was proposed to simulate the features and understand the topology of WMNs.

\section{References}

[1] Ma, X.H., Xu, L. and Min, G.Y. (2013) Congestion Control Based on Cross-layer Game Optimization in Wireless Mesh Networks. 2013 IEEE 9th International Conference on Mobile Ad-Hoc and Sensor Networks, Dalian, 11-13 December 2013, 41-46. http://dx.doi.org/10.1109/msn.2013.49

[2] Benyamina, D., Hafid, A. and Gendreau, M. (2012) Wireless Mesh Networks Design—A Survey. IEEE Communications Surveys \& Tutorials, 14.

[3] Arezoomand, A.S. and Pourmina, M. (2010) Prolonging Network Operation Lifetime with New Maximum Battery Capacity Routing in Wireless Mesh Network. 2nd International Conference on Computer and Automation Engineering (ICCAE), 4.

[4] Huang, H.W., Li, P., Guo, S. and Zhuang, W.H. (2015) Software-Defined Wireless Mesh Networks: Architecture and Traffic Orchestration.

[5] Li, J. (2013) A Cross-Layer Routing Optimization Method in Wireless Mesh Network.

[6] Gupta, B.K., Acharya, B.M. and Mishra, M. K. (2009) Optimization of Routing Algorithm in Wireless Mesh Networks.

[7] Jacquet, P., et al. (2001) Optimized Link State Routing Protocol for Ad Hoc Networks. Proceedings of IEEE INMIC, 62-68. http://dx.doi.org/10.1109/inmic.2001.995315

[8] Akyildiz, I.F. and Wang, X.D. (2005) A Survey on Wireless Mesh Networks. IEEE Radio Communications, September 2005.

[9] Seo, Y., Lim, K.-W., Ko, Y.-B., Song, Y. and Park, S. (2012) Adaptive Wireless Mesh Networks Architecture Based on IEEE 802.11s for Public Surveillance. 9th Annual IEEE Communications Society Conference on Sensor, Mesh and Ad Hoc Communications and Networks (SECON), Seoul, 18-21 June 2012, 65-67. http://dx.doi.org/10.1109/SECON.2012.6275842

[10] Xu, Y.H.Z., Chin, K.-W., Raad, R. and Soh, S. (2016) A Novel Distributed Max Weight Link Scheduler for MultiTransmit/Receive Wireless Mesh Networks. IEEE Transactions on Vehicular Technology.

[11] Yang, C.K., Huang, L.S., Wang, X.L., Xu, H.L. and Leng, B. (2015) (Invited) Popularity-Based Content Replication Scheme for Wireless Mesh Network. 